draft mmm00 8/12/96

\title{
Quantum Computation and Spin Physics
}

\author{
David P. DiVincenzo \\ IBM Research Division \\ Thomas J. Watson Research Center \\ P. O. Box 218 \\ Yorktown Heights, NY 10598 USA
}

(August 28, 2018)

\begin{abstract}
A brief review is given of the physical implementation of quantum computation within spin systems or other two-state quantum systems. The importance of the controlled-NOT or quantum XOR gate as the fundamental primitive operation of quantum logic is emphasized. Recent developments in the use of quantum entanglement to built error-robust quantum states, and the simplest protocol for quantum error correction, are discussed.

1996 PACS: 03.65.Bz, 07.05.Bx, 89.80.+h, 02.70.Rw
\end{abstract}

Typeset using REVTEX 


\section{QUANTUM GATES AND QUANTUM SPINS}

I will give here a very brief summary here of the current activities in the field of quantum computation, particularly some brand-new developments on the preservation of quantum coherence in a noisy environment which may be of interest to those working on the quantum physics of spin systems. It is an awkward story to tell at this point, since much of it is in the process of creation as I write, and I cannot pretend that we have a coherent picture of the whole at the moment. But even the incomplete story is, I think, of some considerable interest.

I will begin with some very basic points about quantum information, which I suspect that many of you have seen before. (There are a number of excellent reviews available, see [1]3.) Suppose I imagine that I use the states of one single, individual $s=1 / 2$ spin to represent the state of a bit; why not? (Of course, there are plenty of practical reasons why not, but I will proceed anyway.) I have to choose a data representation, but there is a very obvious one: the spin-up state $|\uparrow\rangle$ may represent the logical FALSE or zero state $|0\rangle$, and the spin-down state $|\downarrow\rangle$ may represent the TRUE or one state $|1\rangle$. Now, the state of a whole register may be represented by the multi-spin state, say,

$$
\Psi=|001010111 \ldots\rangle
$$

This should already be a big pill to swallow for those of us in magnetic physics; how do you put a system with many spins into that specific wavefunction, and how do you make it stay there? It just isn't done. Well, it ordinarily isn't done; but the remarkable thing is that there are some areas of physics, like atomic physics experiments on ions in vacuum traps [4], where such multi-spin wavefunctions can be manufactured and stabilized. There is nothing in principle to forbid it in solid-state or magnetic physics either, but it is rather exotic.

Let me give an idea of how such multibit states $\Psi$ might be produced in a solid state setting. There will be a lot of "suppose"s in this description, but if you don't like my supposes, you may think up others for yourself. There is one particular multibit state which 
is easy to understand, and is in many contexts easy to manufacture: the state of all zeros, $|000 \ldots\rangle$. We may get this by first putting the spins in a strong enough magnetic field, and then letting them cool down. In fact the all-zero state is a good starting state for all quantum computation. How, then, does one get all the other bit states? In the atomic physics realizations, and perhaps someday in the solid state realizations too, this can be done by manipulating the spins spectroscopically [2]. Suppose that we can illuminate the spins with radiation which is tuned to the resonant frequency connecting the lower, spin-down state with the upper spin up state. Further, suppose (and this is the hard "suppose") this can be done in a perfectly selective fashion, that is, where only one particular spin is in resonance. If you complain that this is impossible, I refer you to the atomic physicists, who are busy doing it. One may imagine doing this by focusing the radiation on a particular spin (if it is being done optically), by applying some magnetic field gradient so that the resonant frequency of some spin is uniquely shifted (this may perhaps be doable with magnetic force microscopy), or by arranging that the selected spin have a different chemical or crystallographic environment (but this is hard to do in a general way for any spin in a large collection).

If this selectivity has been achieved, then the idea is that the radiation causes a controlled time-evolution of the spin's state; in particular, it induces a Rabi oscillation between the lower and upper states of the two-level system. (Not coincidentally, we use the language appropriate for coherent spectroscopy.) If we allow the system to undergo half of a Rabi oscillation period - another jargon for this would be that we have applied a $180^{\circ}$ tipping pulse to the state - then the state of that spin (suppose it is spin number 1) has been flipped:

$$
\Psi=|100 \ldots\rangle
$$

Note that this process is perfectly reversible; if we subject the system to another half-period of Rabi oscillation, it will return to the original state.

So far, this discussion has not used the "quantumness" of the spin states at all; the states discussed above have a one-to-one correspondence to the states in an ordinary computer 
register. If this were all that there was to it, quantum computing would be singularly uninteresting, it would merely be a massively difficult way of mimicking the states of an ordinary computer. What makes things interesting is that one can concoct quantum states which have no analog on a classical machine. Consider what happens if the resonant radiation illuminating spin number 1 is only left on for one-quarter of a Rabi oscillation (corresponding to a $90^{\circ}$ tipping pulse). Then the state of the system evolves into

$$
\Psi=\frac{1}{\sqrt{2}}|000 \ldots\rangle+\frac{1}{\sqrt{2}}|100 \ldots\rangle
$$

a coherent superposition of two bit states. Obviously, the memory of an ordinary computer cannot be placed into a superposition of values; we often remind ourselves of this important difference by using the term qubit rather than bit to refer to this object. Of course, the computer may be programmed to represent such superpositions, but this is very different from the computer memory being in this superposition. Also, this "software" approach is massively inefficient - it is well known that it takes computer resources proportional to $\exp (n)$ to represent an $n$-bit quantum state. It is the possibility of making such superposition states that lends quantum computers their great potential power, permitting them to do parallel computations that make certain problems, like prime factorization, solvable in totally new ways [5]. I will say no more about this here, but refer the reader to various excellent writeups of these results.

But I do want to say something about another obvious question that this brings up: how do you do logic, how do you implement logic gates, which act on the states of these spins? It turns out that besides the one-bit rotations discussed above, only one other gate, a two-bit logic gate which is variously known as the controlled-NOT gate or the quantum XOR gate, is the only additional ingredient which is needed to do logic on quantum states [6]. The controlled-NOT gate has a very classical description: it flips the state of one bit (the target bit) contingent upon the state of a second (control bit). That is, it performs the following transformation on the four possible initial states of two bits: 


$$
\begin{aligned}
& 00 \rightarrow 00 \\
& 01 \rightarrow 01 \\
& 10 \rightarrow 11 \\
& 11 \rightarrow 10
\end{aligned}
$$

Here the first bit is the control, the second is the target. Fig. 1 shows the conventional gate symbol for this operation.

While the controlled-NOT's specification is classical, its implementation cannot be, for it must act on quantum bits, and it must therefore preserve any quantum superpositions with which it is presented, so that, for example, the controlled NOT must successfully perform the following transformation of a superposed input:

$$
a|00\rangle+b|11\rangle \rightarrow a|00\rangle+b|10\rangle
$$

That is, each member of the quantum superposition must be transformed according to the classical rule for the gate.

It will not surprise the reader that implementing this gate will take a few more "suppose"s. The main additional ingredient which is required is an interaction between the spins, that is, a non-zero matrix element of the Hamiltonian coupling them. One may imagine a system which is engineered so that this interaction is turned on temporarily when the gate needs to act (this is the basis of some other proposals I have made using magnetic force microscopy [2,7]; alternatively, fixed interactions, provided they have special, simple forms, will also suffice. For the multi-spin systems, an Ising-like interaction, one in which the coupling interaction only involves the $\sigma_{z}$ Pauli matrix:

$$
H_{\text {int }}=J \sigma_{z}^{i} \sigma_{z}^{j}
$$

is satisfactory. One important reason why this special form of the interaction Hamiltonian is desirable is that all the classical bit states (e.g., |00101011... $)$ are eigenstates of this interaction, and so there is no undesirable time evolution of the quantum states. However, a time evolution corresponding to the controlled-NOT can be induced in this system, if again 
we suppose that we can illuminate the system with radiation which is focussed on just the target spin for the controlled-NOT. Because of the interaction, the transition frequencies depend on the joint state of the two particles. In particular, the $|00\rangle \rightarrow|01\rangle$ transition is at energy $E_{Z}+J$, while the transition energy for $|10\rangle \rightarrow|11\rangle$ has energy $E_{Z}-J$, where $E_{Z}$ is the Zeeman splitting caused by a uniform magnetic field. Because of the shift caused by $J$, the radiation can be tuned so as to be in resonance with one transition and not the other. Except for being specific to one unique spin, the use of such a splitting which is produced by another, coupled quantum system is the common basis of all of double-resonance spectroscopy. It also suffices, if one-half of a Rabi oscillation is induced, to perform essentially the desired controlled-NOT operation. (The phases of the induced transition require a careful discussion, which has been given elsewhere [8]. This discussion does not change the essential features of the argument given here.)

\section{NOISY QUANTUM SYSTEMS AND ERROR CORRECTION}

I have briefly indicated above the kinds of quantum-state manipulations that are necessary in order to perform quantum computations. I now wish to touch upon some of the highlights of an area of intense interest in quantum computation theory now, an area which is absorbing my attention and the attention of many other workers in the field. This is the subject of "quantum error correction", which involves asking the question, how imprecisely can the above operations be performed and still be useful for quantum computation?

Imprecision in the manipulation of quantum systems comes in two apparently very different forms, which however can really be treated in the same way in quantum error correction. They are "decoherence" and "mis-rotation". Decoherence refers to what happens to the quantum system in the presence of an environment; I would like to make a few comments about the mathematics which we use to describe this situation. When a quantum system is not interacting with its environment, its wavefunction $\Psi$ evolves in time according to some unitary time evolution operator $U$ : 


$$
\Psi_{\text {final }}=U \Psi_{\text {initial }} .
$$

A completely equivalent description is that the system's density matrix $\rho=|\Psi\rangle\langle\Psi|$ evolves as

$$
\rho_{\text {final }}=U \rho_{\text {initial }} U^{\dagger} .
$$

It is unnecessary to use the density-matrix description for an isolated quantum system, it is necessary for an open quantum system which is dephasing by interacting with its environment. In this case there is a description of the time evolution which is analogous to Eq. (8), which can in general be written

$$
\rho_{\text {final }}=\sum_{i} A_{i} \rho_{\text {initial }} A_{i}^{\dagger} .
$$

The set of matrices $\left\{A_{i}\right\}$ together make up what is known as a superoperator. The superoperator description of noisy quantum systems is not widely used, but it should be; it is a concise and precise description of the quantum environment, and its parameters may be determined by measurement. (I highly recommend the paper of Schumacher [9] as a compendium of properties of superoperators.)

We will see various examples of superoperator descriptions of the noisy environment below, but I would first like to mention a very important example of one, which is known as the "depolarizing channel" [10]. To call an environment a "channel" borrows some notation from the theory of (quantum) communications, where a qubit is deemed to be subjected to noise during the time that it is resident in the "channel", in motion from place to place; this is only a matter of nomenclature. The superoperator for the depolarizing channel is specified by the four matrices:

$$
\begin{gathered}
A_{0}=\sqrt{f}\left(\begin{array}{ll}
1 & 0 \\
0 & 1
\end{array}\right) \quad A_{1}=\sqrt{\frac{1-f}{3}}\left(\begin{array}{rr}
1 & 0 \\
0 & -1
\end{array}\right) \\
A_{2}=\sqrt{\frac{1-f}{3}}\left(\begin{array}{ll}
0 & 1 \\
1 & 0
\end{array}\right) \quad A_{3}=\sqrt{\frac{1-f}{3}}\left(\begin{array}{rr}
0 & 1 \\
-1 & 0
\end{array}\right)
\end{gathered}
$$


The effect of this channel (or superoperator) is to leave the quantum state alone with probability $f$, and to rotate it about one of three orthogonal axes by $90^{\circ}$ (I am using a "Bloch sphere" notation here) with probability $(1-f) / 3$. Another equivalent description is that it involves leaving the quantum state alone with probability $(4 f-1) / 3$, and, with probability $(2-4 f) / 3$, replacing it with a completely random quantum state. $((2-4 f) / 3$ is referred to as the depolarization fraction in this case.)

Now, after all this, let me return to the question of imprecision of quantum gate operations. Suppose that because of the nature of your experimental setup, when you are trying to make a gate corresponding to the unitary transformation $U\left(\left\{\theta_{0}\right\}\right)$ with some specified rotation angles $\left\{\theta_{0}\right\}$ you will actually produce one of a range of unitary transformations drawn from a probability distribution $p\left(\left\{\theta_{0}\right\}\right)$. Then the new density matrix after operation of this partially known gate is

$$
\rho_{\text {final }}=\int d\{\theta\} p(\{\theta\}) U(\{\theta\}) \rho_{\text {initial }} U^{\dagger}(\{\theta\})
$$

There is clearly a correspondence between the matrices $\sqrt{p(\{\theta\})} U(\{\theta\})$ and the matrices $A_{i}$ of the superoperator above. The correspondence may be made even more exact with a Gram-Schmitt transformation which converts the continuous set of matrices $\sqrt{p(\{\theta\})} U(\{\theta\})$ into a discrete set - just four of them, in fact, when the dimension of $\rho$ is 2 , just as in the depolarizing-channel example above. The conclusion of this is that uncertainty in the unitary transformation performed by a quantum gate is really indistinguishable from the situation in which first the gate is performed perfectly, and then the qubits interact with some noisy environment. This effective environment can have some strange properties; for instance, for the case of an uncertain two-bit gate, the effective noise may correspond to some correlated interaction of the two qubits with the effective environment, rather than the common case of a real environment in which the two qubits interact independently with their environments (we will see in a moment a superoperator description of such a situation).

So, decoherence and misrotations are types of errors in quantum computation which can be placed on exactly the same footing. Now, we come to the question of how one is 
to correct for such errors. In less than a year preceding this writing, the theory of errorcorrection of quantum systems has gone from nearly nothing to a fantastically developed and mathematically sophisticated edifice [11]. This monumental theoretical development also has, in my opinion, the potential to influence experimental quantum physics (not just quantum computation) in many profound ways.

Having said this, I must humbly admit that I will give here only a few small morsels of the feast which appears to await us. I think that theorists are still struggling to explain all that they have discovered about quantum error correction, and an attempt at a full explanation of current developments would come across as abstruse and obscure, at least if I were to write it. But in offering up only morsels I have the option of explaining just the most elementary parts of the ideas, and also the ones which are likely to most readily testable within the current experimental art.

In this I hope to bring home a rather paradoxical idea about entangled states. Entangled states, famous in quantum physics in the Einstein-Podolsky-Rosen paradox [12], Bell's inequalities [13], and so forth, are quantum states of two (or, especially for the purposes of quantum error correction, more than two) subsystems, in which the wavefunction of the pair is not a product of the wavefunctions of the individual subsystems. For two spin-1/2 systems, for example, a legal quantum state is

$$
\Phi=|00\rangle+|11\rangle
$$

(Recall that we denote spin down by 0 , spin up by 1.) This state and other related ones are illustrative of the weird non-locality of quantum correlations; when the two spins are spatially separated, we say that the common direction of the two separate spins is perfectly correlated, even while each separately has a completely indeterminate direction. (We will not be so much interested, in the examples from quantum error correction theory, of the weirdness attendant on the remote physical separation of these components. We will be content, in fact, for all the components to be in close physical proximity. The mathematical weirdness persists.) The EPR-type state above is also the smallest example of a "macroscopic" superposition 
state; the really macroscopic version of this is the famous "Schrodinger cat" state [14, $\mid$ alive $\rangle+\mid$ dead $\rangle$.

Both of the states illustrated above are considered to be very fragile quantum states; a small amount of interaction with the environment changes the state into a classical mixture in which all of the spooky quantum correlations have been lost. But, the paradoxical message from quantum error correction theory is that while entangled states do fall apart when subjected to noise from the environment, they can be designed to fall apart in such a way that the quantum correlations are not irretrievably lost, and so that the original quantum state is in fact recoverable (via a quantum computation, of course).

With this run-up, I will finally give my one simple worked-through example of quantum error correction which illustrates all of the assertions I have made below. Suppose the object is simply to store (or perhaps transmit) a quantum bit, which may be in some arbitrary state

$$
\Psi=a|0\rangle+b|1\rangle
$$

i.e., with arbitrary complex coefficients $a$ and $b$ (satisfying the normalization condition). Now, I imagine that this qubit is subjected to a (toy) environment characterized by the two matrices

$$
\begin{gathered}
A_{1}=\sqrt{f}\left(\begin{array}{ll}
1 & 0 \\
0 & 1
\end{array}\right) \\
A_{2}=\sqrt{1-f}\left(\begin{array}{ll}
0 & 1 \\
1 & 0
\end{array}\right)
\end{gathered}
$$

$f$ is a characterization of the fidelity of this "channel", with $f=1$ corresponding to the noiseless case. It is a toy channel because it does not correspond the typical real noisy channel; in words one can say that with probability $f$ it leaves the qubit alone, and with probability $1-f$ is flips 0 to 1 and vice versa. As one can see, it is a stripped-down version of the depolarizing channel above (for some comments about the error correction for that 
case, see the very end); also, it bears some resemblance to a "decay" channel, which is a very real model for some applications in quantum optics [15], where the superoperator is specified by

$$
\begin{gathered}
A_{1}=\left(\begin{array}{cc}
1 & 0 \\
0 & \sqrt{f}
\end{array}\right) \\
A_{2}=\left(\begin{array}{rr}
0 & \sqrt{1-f} \\
0 & 0
\end{array}\right)
\end{gathered}
$$

where typically $f$ is taken to be an exponential function of time: $f(t)=e^{-t / \tau}$, with some characteristic decay time $\tau$. Despite their resemblances, though, the decay channel takes rather more work to error-correct than my toy channel, and I will be sticking with that here.

So, enough of the environment. What does it do to my poor qubit? Of course, that depends on what $a$ and $b$ are; in general, though, I will characterize the quality of the degraded qubit by a fidelity, an overlap between the original state and the state after interaction with environment. If the density matrix specifying this final state is $\rho$, then the fidelity $F$ is simply

$$
F=\langle\Psi|\rho| \Psi\rangle
$$

$F$ is obviously a function of $a$ and $b$, as well as the channel parameter $f(t)$ (or just $t$ ). The right thing to do is to minimize $F$ over $a$ and $b$, or take averages over their expected distribution, but I will just pick particular $a$ 's and b's and discuss the behavior of $F$ with $t$.

It is trivial to see that if $a=1$ in Eq. 13, then

$$
F(t)=e^{-t / \tau}
$$

i.e., the fidelity of the state falls exponentially. Now we will see how the tools of quantum error correction can be used to improve upon this. The first idea is that we replace the bare qubit with a coded qubit. In this case a good way to code the qubit is to represent the 
$|0\rangle$ state by the three-qubit state $|000\rangle$, and the $|1\rangle$ by the state $|111\rangle$; thus, the qubit is encoded in the three-qubit entangled state

$$
\Psi=a|000\rangle+b|111\rangle
$$

This state is a close relative of the "Greenberger-Horne-Zeilinger" state [16], which has long been discussed in scenarios of multi-particle entanglement and quantum non-locality. Now we will see how this state can be used to more-robustly (i.e., with higher fidelity) preserve the qubit against the ravages of the noisy channel.

First, we imagine that each of the three qubits in the state above is subjected to an independent copy of the toy channel introduced above. This means that the operators which make up the complete superoperator will have a direct-product form:

$$
A_{i j k}=A_{i}^{(1)} \otimes A_{j}^{(2)} \otimes A_{k}^{(3)}
$$

If we compute the fidelity after this superoperator has been applied, just using the same formula as above, we find that it is actually less than for the uncoded qubit, in fact, again for the case $a=1$ in Eq. (13):

$$
F(t)=e^{-3 t / \tau}
$$

That is, the fidelity falls three times faster than for the unencoded state; so, entangled states are more delicate than unentangled ones.

We seem to be not at all closer to our goal of making the qubit last longer. But the key is that, while the noisy entangled state has lower fidelity, it is more recoverable. To see what this means, suppose that the toy channel had only acted on one of the qubits, and that the qubit had been flipped. In this case, the state of the system may have been turned into

$$
\Psi=a|100\rangle+b|011\rangle
$$

(The full density matrix of the state after it has interacted with the channel is a statistical mixture of such flipped states with appropriate probability weights.) Now, this state has 
zero fidelity, because it is orthogonal to the original coded qubit state. However, unlike the uncoded qubit, the coded state can be processed, by an error correction step, to recover the original qubit exactly. (Details of the example which I show now has been presented by A. M. Steane in Ref. [11].)

This error correction proceeds in the following fashion: We perform a sequence of incomplete von Neumann measurements on the state. The first one asks the question, "are qubits 1 and 2 in the same state, or not?" To "ask" this question of the quantum state, we perform a quantum computation on it, using (not surprisingly) the quantum XOR or controlled NOT operation which I emphasized in the first part as the fundamental gate of quantum computation. Here's how it works (referring to Fig. 2): introduce a fourth, ancillary bit, initially setting it to $|0\rangle$. Then do two XORs, the first with qubit 1 as the source bit, the second with qubit 2 as the source; in both cases, the target is the ancilla qubit 4 . If qubits 1 and 2 are in the state 00, then bit 4 is untouched; if they are in the state 11, then qubit 4 is flipped twice, and so is again in its original state $|0\rangle$. On the other hand, if the source state is 01 or 10 , the ancilla bit gets flipped just once, and ends up in the state $|1\rangle$. Then, a measurement of qubit 4 is exactly the incomplete measurement we have specified above. It is crucial to recognize that this measurement obtains information about the entangled state without disturbing it. This is only possible because of the special form of the entangled state; the two parts of the state $a|100\rangle$ and $b|011\rangle$ give the same measurement outcome, so their quantum superposition is undisturbed.

Just one other measurement of this sort, on qubits 1 and 3, suffices to determine exactly which of the three qubits was disturbed by the channel. Referring to Fig. 2, it is easy to see that if $M_{1}=M_{2}=0$, then none of the three qubits is disturbed, if $M_{1}=1$ and $M_{2}=0$, then it must have been qubit 1 that had been flipped, if $M_{1}=0$ and $M_{2}=1$ then the disturbed qubit must have been number 3 , and if $M_{1}=M_{2}=1$, the number 2 is the disturbed one. Since the disturbance caused by the channel is completely known, and the form of the entangled state is undisturbed by these measurements, A final quantum gate performing a flip or NOT on the affected qubit serves to restore the state exactly to its 
original form.

Of course, this error correction procedure, just like any classical one, will be fooled if too many qubits have been flipped. The procedure above will be fooled if a two-bit error is caused by interaction with the environment. But at early times, when the probability of a single qubit-flip $(1-f)$ is small, the probability of two qubit flips is much smaller, of order $(1-f)^{2}$. Therefore, if the error correction is performed after a sufficiently brief interaction with the environment, the error-corrected state will have higher fidelity than the bare qubit state. Fig. 3 shows these fidelities for the example above; the coded state has higher fidelity up until a time corresponding to $f=1 / 2$.

The crucial feature of these curves, though, is that the early-time fidelity is very high, going like $1-c(1-f)^{2}$. This means that if error correction is done often enough (more frequently than the natural decay time), the fidelity may be kept high for much longer than would be possible for the uncoded qubit in contact with its environment. This is the essence of the error-robustness of entangled states.

I have finished the tale I wanted to tell, but it is really just the tip of the iceberg on the story of quantum error correction, which I and a number of other workers in the field have been intensely studying. First, we know of encodings that will protect states, in the same way just outlined, from more general types of interaction with its environment. In particular, we [10] and others found a code that would protect against interaction with the depolarizing channel above; five-bit entangled states are required for this. This code also works for the quantum-optics decay channel, although it is not known whether there might be more efficient codes for dealing with this particular case. There are also large families of codes which are capable of detecting and correcting more than one-bit errors [11]. This means that the short-time corrected fidelity is better than the example given above, going like $F(t)=1-c(1-f)^{t+1}$, where $t$ is the number of errors corrected. Larger-size entangled states, and more extensive quantum computation for error correction, are required for this. Still, there is a recent idea of Shor [17], being actively investigated by a number of workers, that large-block codes, correcting many errors, may be optimal for performing long quantum 
computations reliably. There are, I believe, several years of theoretical work still ahead of us to find the ultimate capability of quantum computation, particularly in the presence of noise. 


\section{REFERENCES}

[1] C. H. Bennett, "Quantum Information and Computation", Physics Today, October, 1996, p. 27.

[2] D. P. DiVincenzo, "Quantum Computation” Science 270, 255 (1996).

[3] A. Ekert and R. Jozsa, "Quantum computation and Shor's factoring algorithm", Rev. Mod. Phys., to be published (1996).

[4] C. Monroe, D. M. Meekhof, B. E. King, W. M. Itano, and D. J. Wineland, "Demonstration of a Fundamental Quantum Logic Gate," Phys. Rev. Lett. 75, 471 (1995).

[5] P. W. Shor, "Algorithms for quantum computation: discrete log and factoring", Proceedings of the 35th IEEE Symposium on the Foundations of Computer Science, (IEEE Computer Society Press, New York, 1994), p. 124.

[6] A. Barenco, C. H. Bennett, R. Cleve, D. P. DiVincenzo, N. Margolus, P. Shor, T. Sleator, J. A. Smolin and H. Weinfurter, "Elementary gates for quantum computation", Phys. Rev. A 52, 3457 (1995).

[7] D. P. DiVincenzo, "Two-bit gates are universal for quantum computation", Phys. Rev. A 50, 1015 (1995).

[8] R. Cleve and D. P. DiVincenzo, Schumacher's quantum data compression as a quantum computation," Report No. quant-ph/9603009.

[9] B. Schumacher, "Sending quantum entanglement through noisy channels," Report No. quant-ph/9604023.

[10] C. H. Bennett, D. P. DiVincenzo, J. A. Smolin, W. K. Wootters, "Mixed state entanglement and quantum error-correcting codes," Report No. quant-ph/9604024.

[11] For a small sampling of this edifice, see A. R. Calderbank, E. M. Rains, P. W. Shor, and N. J. A. Sloane, "Quantum error correction and orthogonal geometry," Report 
No. quant-ph/9605005; "Quantum error correction via codes over GF(4)," Report No. quant-ph/9608006; R. Laflamme, C. Miquel, J. P. Paz, and W. H. Zurek, "Perfect quantum error correcting code", Phys. Rev. Lett. 77, 198 (1996); A. M. Steane, "Error correcting codes in quantum theory", Phys. Rev. Lett. 77, 793 (1996); D. P. DiVincenzo and P. W. Shor, "Fault-Tolerant Error Correction with Efficient Quantum Codes," Report No. quant-ph/9605031.

[12] A. Einstein, B. Podolsky, and N. Rosen, "Can quantum-mechanical description of physical reality be considered complete?", Phys. Rev. 47, 777 (1935). Reprinted in Quantum Theory and Measurement (J.A. Wheeler and W.Z. Zurek, eds, Princeton University Press, 1983.

[13] J. S. Bell, "On the Einstein-Podolsky-Rosen paradox." Physics 1, 195 (1964). Reprinted in J. S. Bell, Speakable and Unspeakable in Quantum Mechanics (Cambridge University Press, 1987), p. 14.

[14] E. Schrodinger, "The present situation in quantum mechanics," Naturwissenschaften 23, 807, 823, 844 (1935); translated in Quantum Theory and Measurement, eds. J. A. Wheeler and W. H. Zurek (Princeton University, Princeton, NJ, 1983).

[15] I.L. Chuang and R. Laflamme, "Quantum Error Correction by Coding," Report No. quant-ph/9511003.

[16] N. D. Mermin, Physics Today 43 (June 1990) p. 9.

[17] P. W. Shor, "Fault-tolerant quantum computation," Report No. quant-ph/9605011. 


\section{FIGURES}

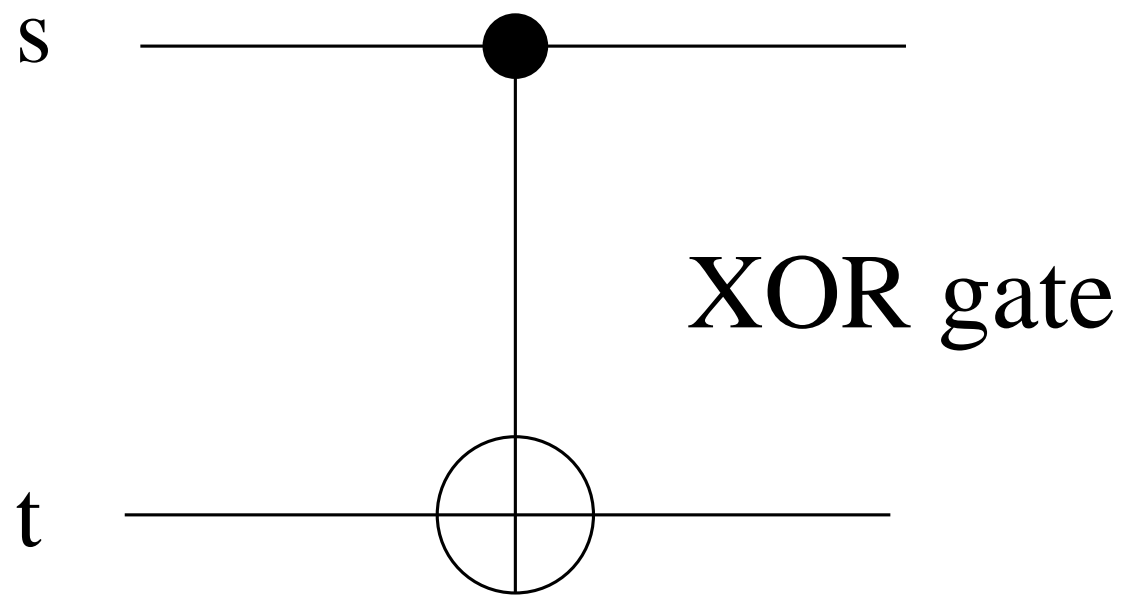

\section{Fig. 1 DiVincenzo}

FIG. 1. The gate symbol for the controlled-NOT or XOR gate, the fundamental two-bit gate of quantum logic. The upper line denotes the source qubit, and the lower line the target qubit. 


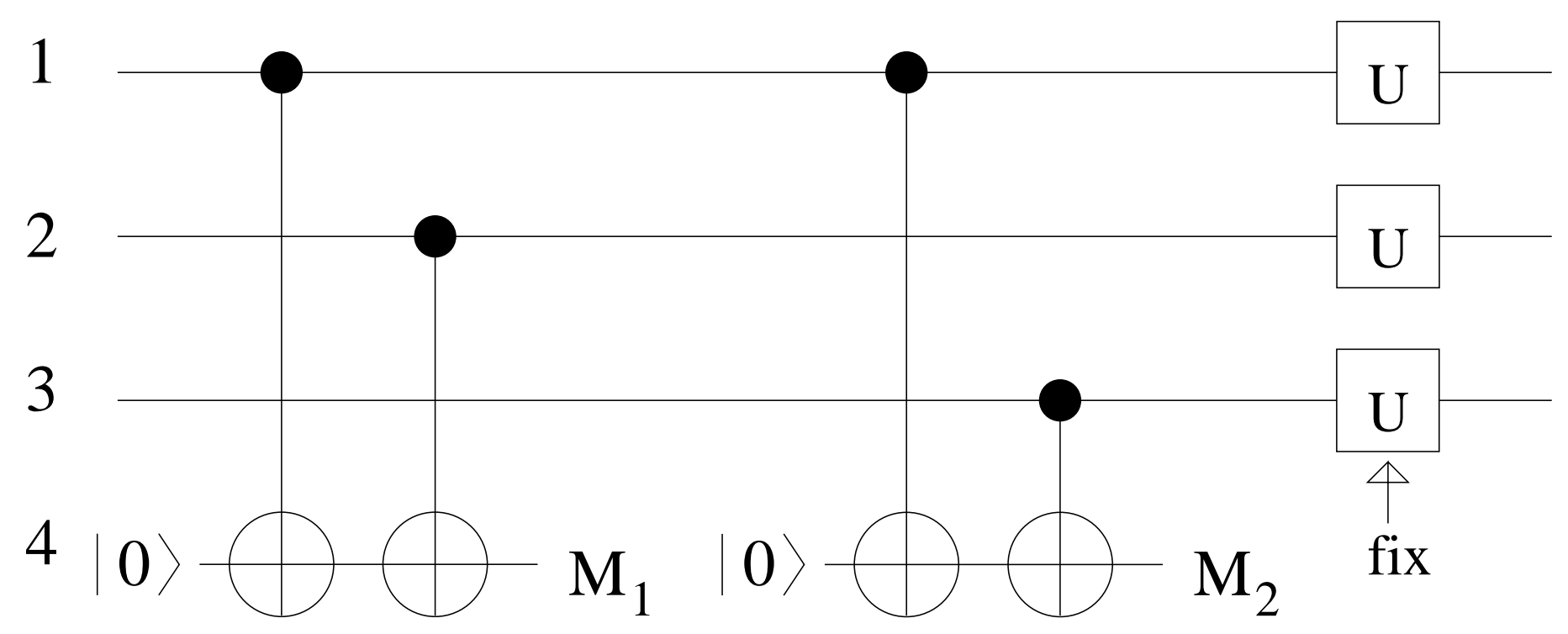

Fig. 2 DiVincenzo

FIG. 2. Quantum network, using XOR gates, one-bit measurements, and a final one-bit rotation, for performing error correction on the entangled code state. 


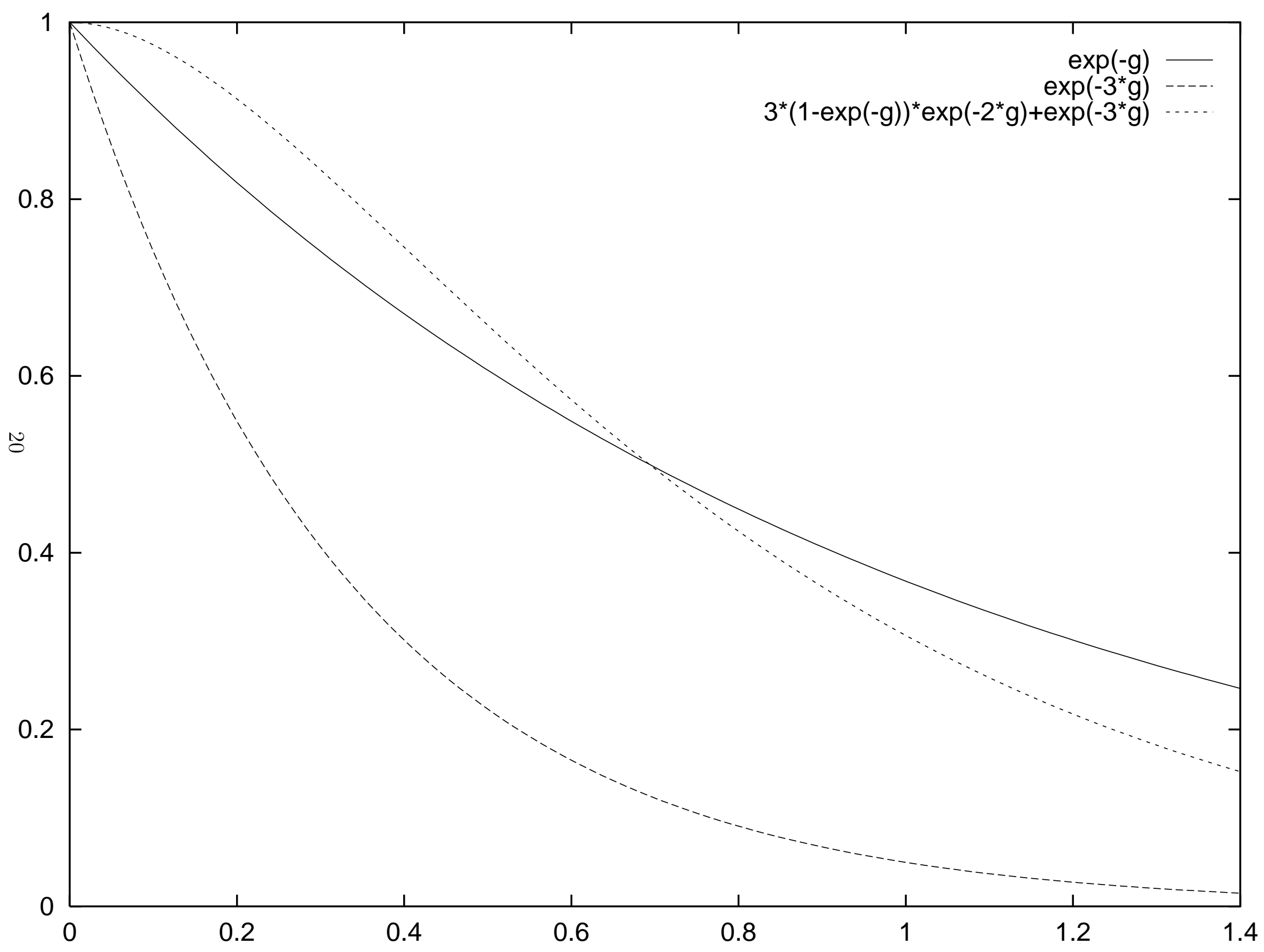


FIG. 3. Fidelity vs. time with and without quantum error correction, as described in the text. Solid line: $f=\exp (-t / \tau)$, fidelity of the unencoded qubit. Dashed line: $f=\exp (-3 t / \tau)$, fidelity of the triple-coded qubit without error correction. Dotted line: $f=3(1-\exp (-t / \tau)) \exp (-2 t / \tau)+\exp (-3 t / \tau)$, the fidelity of the coded state after it has been error-corrected at time $t$. 